УДК 622.276 .342

\title{
ОПТИМИЗАЦИЯ РАССТОЯНИЯ МЕЖДУ СКВАЖИНАМИ СИСТЕМЫ СБОРА БИОГАЗА
}

Куцый Д.В., канд. техн. наук

Институт технической теплофизики НАН Украины, ул. Желябова, 2a, г. Киев, 03680, Украина

Запропоновано підхід до оптимізації відстані між свердловинами, який базується на максимальній ефективності системи збору біогазу і витраті на газозбірній свердловині. 3 використанням цього підходу визначені та проаналізовані значення оптимальної відстані між свердловинами.

Библ. 14, рис. 5.
Предложен подход к оптимизации расстояния между скважинами, который базируется на максимальной эффективности системы сбора биогаза и расходе на газосборной скважине. С использованием этого подхода определены и проанализированы значения оптимального расстояния между скважинами.
The principle of optimization of a distance between wells which is based on the maximum efficiency of landfill gas collection system and the flow rate in the well is offered. With the use of this principle, the values of the optimal distance between wells are determined and analyzed.

Ключевые слова: биогаз, газосборная скважина, оптимальное расстояние, радиус действия, твердые бытовые отходы.

$f-$ функция;

$g-$ расход, кг/с;

$h$ - высота, м;

$X, Y, Z$ - оси координат;

$\gamma$ - коэффициент увеличения расхода, \%;

$\varphi$ - эффективность системы сбора биогаза, \%;

ГС - газосборная скважина;

РДС - радиус действия скважины;

ТБО - твердые бытовые отходы.

\section{Введение}

Использование систем сбора биогаза является общепринятым, а в некоторых странах обязательным техническим решением по дегазации полигонов твердых бытовых отходов (ТБО). Наибольшее распространение среди них получили системы сбора биогаза, состоящие из вертикальных газосборных скважин (ГС), что объясняется возможностью их использования как на действующих, так и на закрытых полигонах ТБО.

Основным параметром, который определяет расположение вертикальных ГС и впоследствии конструкцию системы сбора биогаза, является радиус действия скважины (РДС). Как показывают результаты исследований, РДС зависит от га-

\section{Индексы нижние:}

0 - засыпка;

1 - гравий;

2 - глина;

1well - одна независимая скважина;

2 well - система из двух скважин;

1-2well - одна их системы двух скважин.

gen - образование биогаза;

$j$ - компонент биогаза.

зообразования [1-3, 5-6], проницаемости отходов $[2-3,5,11]$, проницаемости и толщины верхнего покрытия [3], конструкции полигона $[1,6]$, конструктивных и эксплуатационных параметров ГС [3-11], а также других факторов, которые отображают условия определенного полигона ТБО или характеризуют ГС.

Кроме того, РДС зависит от расстояния до соседних скважин системы сбора биогаза, причем эта зависимость сохраняется в условиях разных полигонов ТБО. Очевидно, что для по-вышения эффективности системы сбора биогаза, ГС должны располагаться как можно ближе друг к другу. При этом усиливается их взаимное влияние, а количество биогаза, доступное для сбора в области 
между скважинами, уменьшается.

Изменение эффективности системы сбора биогаза в зависимости от расстояния между скважинами исследовано в [3]. Авторы установили линейное уменьшение эффективности системы сбора биогаза при увеличении расстояния между ГС. С другой стороны, при увеличении расстояния между ГС, как показано в [12], расход биогаза на отдельной скважине увеличивается. Возникает задача определения оптимального расстояния между скважинами, при котором эффективность системы сбора биогаза и расход на ГС достигают своих максимальных значений.

В литературе встречаются несколько подходов к определению оптимального расстояния между скважинами. Так, в [8, 13] оптимальное расстояние между ГС определено из геометрических соображений. Его величина в 1,73 раза больше РДС. В [5] оптимальное расстояние между ГС составляет удвоенный РДС.

Главным недостатком всех подходов, предложенных в $[5,8,13]$ для определения оптимального расстояния между ГС, является отсутствие учета эффективности системы сбора биогаза и расхода на ГС, исследованных в $[3,11]$. Возможно, поэтому и возникли разные подходы в литературе. Учет этих фундаментальных параметров для определения оптимального расстояния между ГС и является целью данной работы.

\section{Методы исследований}

Для определения оптимального расстояния между ГС рассматриваем две целевые функции с двумя переменными. Первая функция зависит от эффективности системы сбора биогаза и расстояния между скважинами, а вторая функция - от расхода на ГС и расстояния между скважинами.

Эффективность системы сбора биогаза определяется как отношение количества биогаза, собранного скважинами, к количеству биогаза, образованного в толще отходов полигона ТБО

$$
\varphi=\frac{\sum_{j=1}^{n} \dot{g}_{2 w e l l, j}}{\sum_{j=1}^{n} \dot{g}_{g e n, j}} 100 \% .
$$

При проектировании системы сбора биогаза эффективность должна стремиться к максимальному значению $100 \%$.
В свою очередь, расход на отдельной ГС зависит от расположения соседних скважин системы сбора биогаза, а его максимальное значение достигается в условиях отсутствия влияния соседних скважин или в условия работы одной скважины [12]. Исходя из этого, расход на ГС можно выразить в относительных величинах, введя понятие коэффициента увеличения расхода

$$
\gamma=\frac{\sum_{j=1}^{n} \dot{g}_{1-2 \text { well }, j}}{\sum_{j=1}^{n} \dot{g}_{1 \text { well }, j}} 100 \% .
$$

Выразив переменную второй целевой функции в относительных величинах, получена универсальная область определения, для которой математически оптимизационную задачу можно записать в виде

$$
\max \left\{\begin{array}{l}
f(\varphi ; r) \\
f(\gamma ; r)
\end{array}, \quad 0<(\varphi ; \gamma)<100 \% .\right.
$$

Решение оптимизационной задачи (3) сводится к нахождению точки, в которой обе целевые функции принимают максимально-доступные значения. Для определения целевых функций и нахождения этой точки использовалась математическая модель, разработанная в [14]. Исходные данные модели полностью соответствовали параметрам свалки, которые приведены в [9].

Используемая математическая модель состоит из уравнений сохранения массы и энергии, описывающих процессы образования и транспорта компонентов биогаза (метан, углекислый газ, азот и кислород) и теплоты в пористой среде отходов. Особенностями модели является учет изменения гидродинамических свойств отходов вследствие их вторичного проседания, динамики газообразования и продолжительности периода складирования отходов, которые отображают условия реального полигона ТБО. Более детально с возможностями модели можно ознакомиться в [14].

Моделирование проводилось с использованием расчетной области, показанной на рис. 1. Область представляет собой элемент полигона ТБО длиной $r$ и высотой $h$. Слева и справа расчетной области располагаются ГС с примыкающими слоями засыпки $r_{0}$, состоящими из гравия 
и глины высотой $h_{1}$ и $h_{2}$ соответственно. Между скважинами находится слой отходов.

Через перфорированный участок ГС, где устанавливается разряжение, происходит отбор биогаза из слоя отходов. При этом поверхность полигона ТБО остается непокрытой, вследствие чего отходы контактирует с атмосферным возду- хом. Здесь при атмосферном давлении и температуре происходит выход биогаза и инфильтрация воздуха, которые сопровождаются теплообменом. Нижняя граница полигона ТБО представлена непроницаемым для потока биогаза уровнем фильтрата, который имеет постоянную температуру.

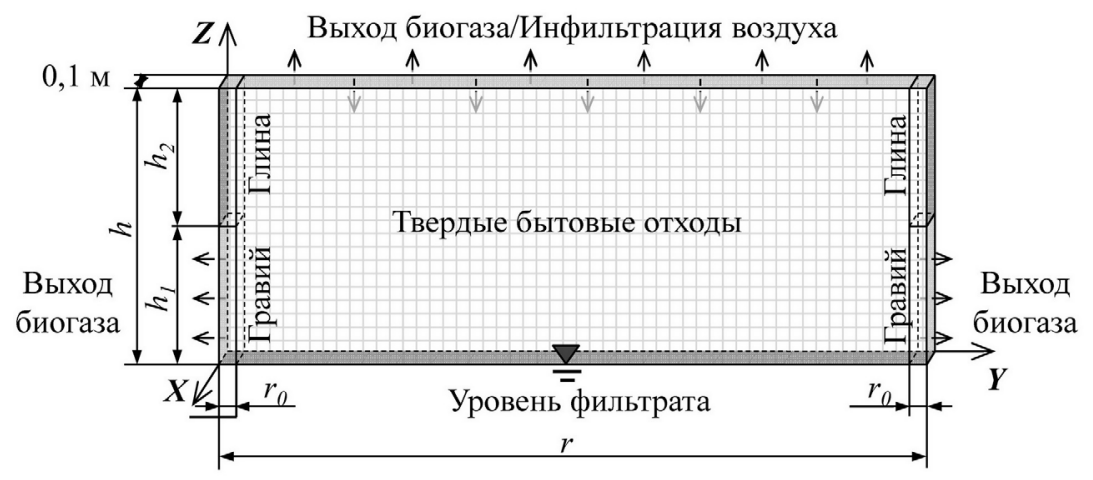

Рис. 1. Расчетная область.

В процессе моделирования изменялась длина расчетной области $r$ и рассчитывались искомые параметры по уравнениям (1) и (2). Максимальная длина области составляла 100 м, которая более чем в два раза превышала РДС. Разряжение на обеих скважинах устанавливалось одинаковым.

\section{Анализ результатов}

Результаты моделирования в виде изменения эффективности системы сбора биогаза и расхода на ГС в зависимости от расстояния между скважинами при постоянном разряжении - 0,5 кПа показаны на рис. 2.

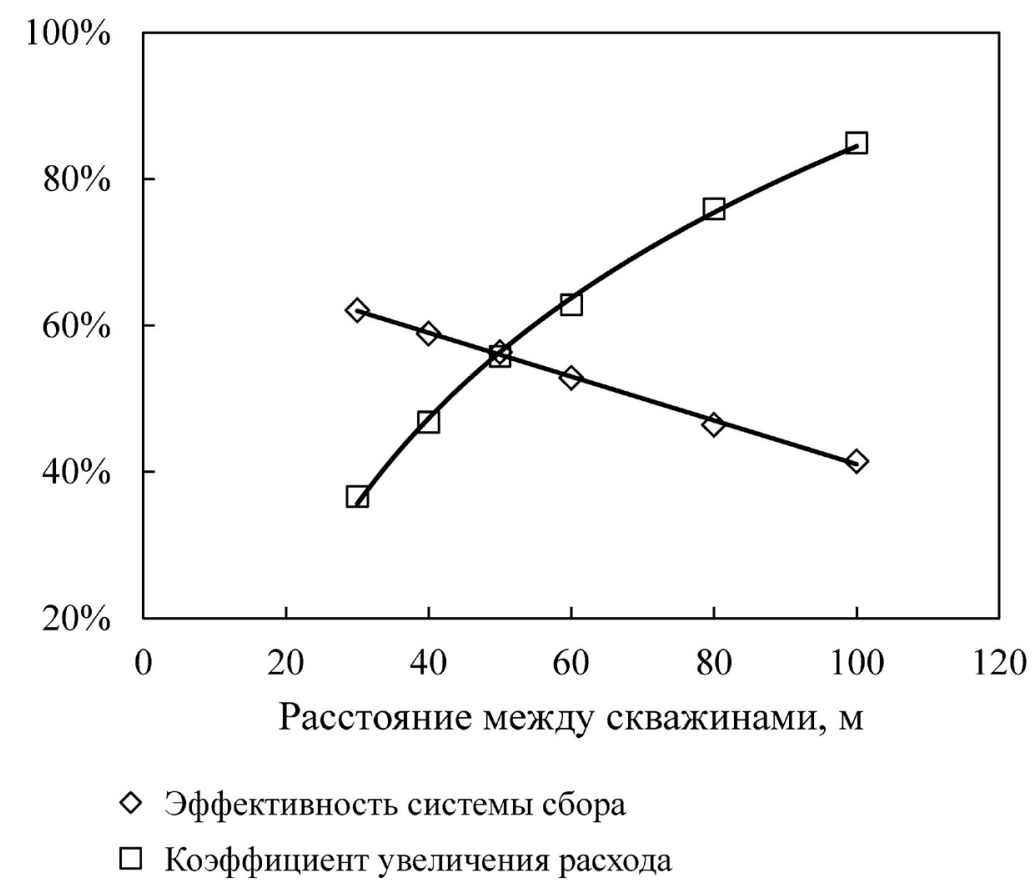

Рис. 2. Зависимость эффективности системы сбора биогаза и расхода на скваэнне от расстояния между скважинами при разряжении - 0,5 кПа. 
Видно, что с увеличением расстояния между скважинами эффективность системы сбора биогаза линейно уменьшается. Это объясняется увеличением области отходов, в которой уменьшается влияние РДС, и все больше биогаза устремляется в вертикальном направлении к выходу в атмосферу через поверхность полигона ТБО. При этом расход на каждой из ГС системы сбора биогаза увеличивается по логарифмической зависимости, обратной зависимости коэффициента уменьшения расхода, полученной в [12]. Увеличение расхода свидетельствует о снижении взаимного влияния РДС друг на друга.

В точке пересечения обеих зависимостей достигается максимальный расход на ГС и максимально-доступная эффективность системы сбора биогаза, которая при этом получается (см. рис. 2). Эта точка соответствует оптимальному расстоянию между скважинами 49,5 м и, как оказывается, представляет собой удвоенный РДС.

Оптимальное расстояние между скважинами изменяется в зависимости от разряжения, как показано на рис. 3. При этом полученная квазилинейная зависимость совпадает с результатами [5].

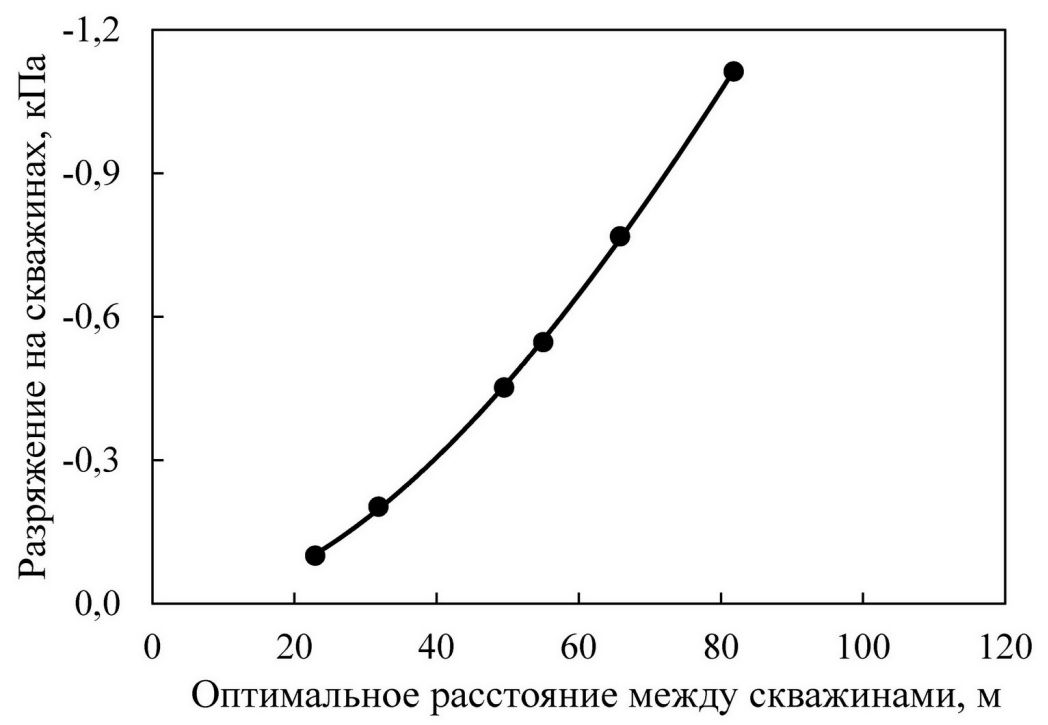

Рис. 3. Зависимость оптимального расстояния между скважинами от разряжения.

Наибольшее оптимальное расстояние между скважинами получается при более глубоком разряжении, что объясняется большим РДС. Однако глубокое разряжение может приводить к развитию инфильтрации воздуха в тело полигона ТБО. В данном случаи (для свалки), инфильтрация активно развивается, при разряжении, превышающем - 0,5 кПа [9]. Поэтому оптимальное расстояние между скважинами ограничивается допустимым разряжением.

При увеличении оптимального расстояния между скважинами вследствие изменения разряжения возрастает и эффективности системы сбора биогаза, характер изменения которой изображен на рис. 4.

Видно, что возрастание эффективности системы сбора биогаза хорошо описывается ло- гарифмической зависимостью. Поэтому значения эффективности системы сбора биогаза в наибольшей мере изменяются при малых оптимальных расстояниях между скважинами. То есть при увеличении оптимального расстояния между скважинами от 30 до 40 м, эффективность системы сбора биогаза возрастает от 36 до 47 \%, а при увеличении оптимального расстояния между скважинами от 70 до 80 м, эффективность системы сбора биогаза возрастает от $71 \%$ до $78 \%$.

\section{Использование результатов}

Полученные результаты использовались при разработке промышленной системы сбора биогаза на полигоне ТБО в г. Ровно. Полигон действует с 1959 года и на сегодняшний день занимает площадь 21,2 га, из которой под отходами занято 
около 17,2 га. Глубина отходов варьируется от 6 до 20 м, а общее количество оценивается порядка 1,5 млн. тон.

На участках полигона глубиной более 10 м могут сооружаться вертикальные ГС. Допустимое разряжение на скважинах принимается 0,5 кПа, обеспечивающее отсутствие инфильтрации воздуха в тело полигона ТБО. При та- ком разряжении оптимальное расстояние между скважинами составляет 50 м (см. рис. 3).

С учетом выбранных параметров выполнено расположение ГС по площади полигона ТБО, которое представлено на рис. 5.

Всего для сбора биогаза на полигоне ТБО рекомендуется соорудить 31 скважину. Все они соединяются с магистральным трубопроводом,

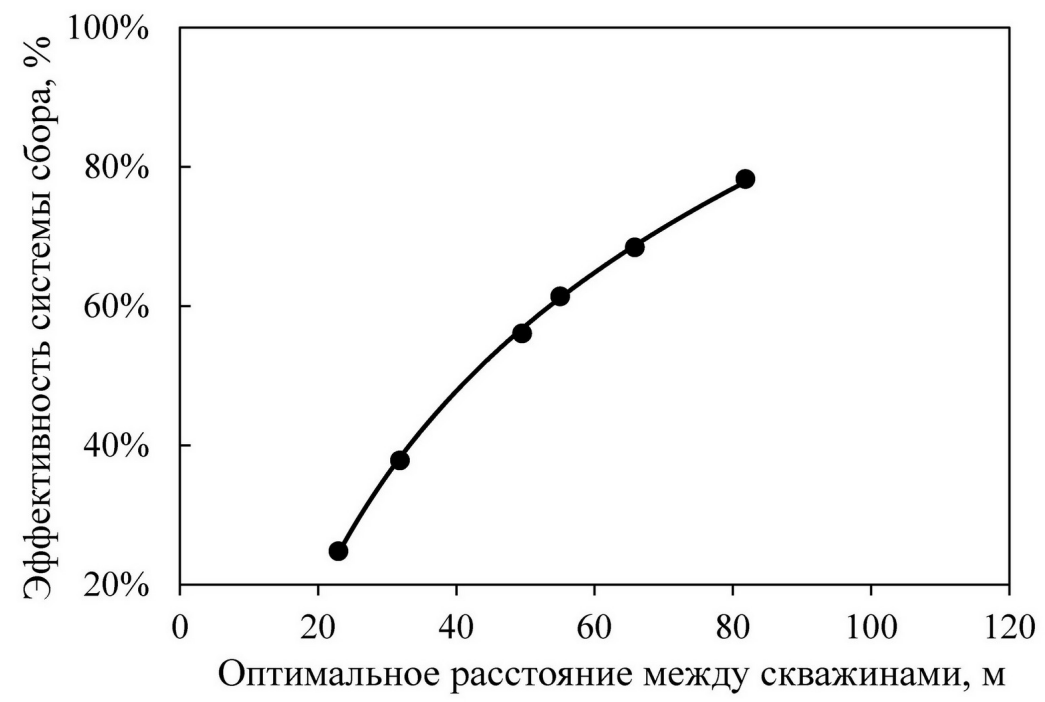

Рис. 4. Зависимость эффективности системы сбора биогаза от оптимального расстояния между скважинами.

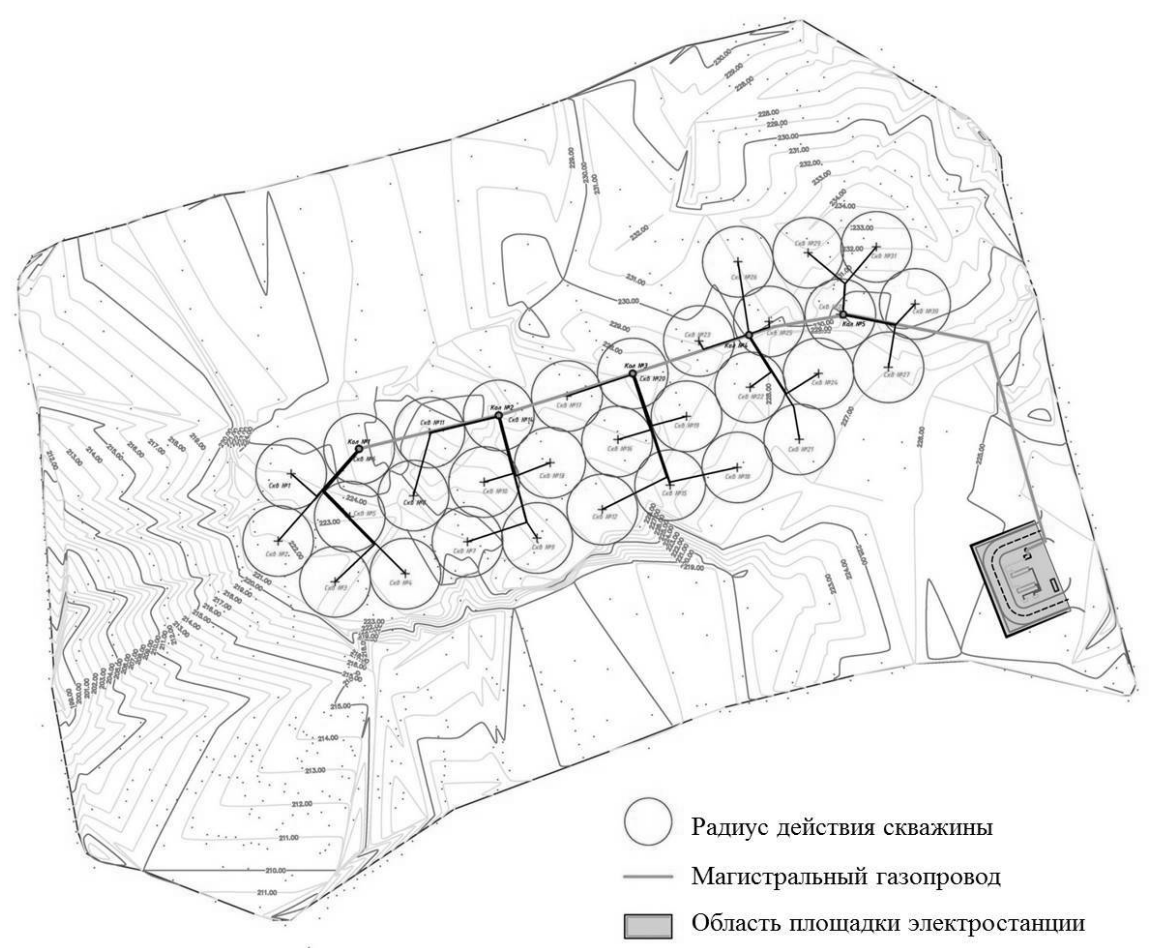

Рис. 5. Эскиз системы сбора биогаза на полигоне ТБО г. Ровно. 
который транспортирует биогаз с тела полигона ТБО на площадку электростанции, расположенную возле въезда (см. рис. 5). В пределах площадки устанавливается оборудование для откачивания и утилизации биогаза с производством электроэнергии, которая подается в сеть.

Общая эффективность разработанной системы сбора биогаза составляет 57 \% (см. рис. 4), при которой в 2015 году обеспечивался расход биогаза на уровне $285 \mathrm{~m}^{3} /$ час. Принимая содержание метана в биогазе $45 \%$, собранного газа будет достаточно для работы генерирующей установки мощностью 500 кВ эл $_{\text {. }}$

\section{Выводы}

Оптимальное расстояние между скважинами системы сбора биогаза равняется удвоенному радиусу действия скважины. Оно увеличивается при увеличении разряжения на скважине, поскольку растет ее радиус действия. Однако это увеличение ограничивается допустимым разряжением, при котором отсутствует инфильтрация воздуха в тело полигона ТБО.

На основании полученных результатов разработана промышленная система сбора биогаза для полигона ТБО в г. Ровно, состоящая из 31 газосборной скважины. Эффективность данной системы составляет $57 \%$, при которой расход биогаза оценивался на уровне $285 \mathrm{~m}^{3} /$ час в 2015 году. Собранного биогаза будет достаточно для работы генерирующей установки мощно-

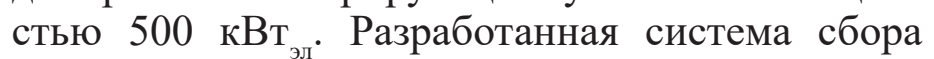
биогаза рекомендуется к внедрению.

\section{ЛИТЕРАТУРА}

1. Vigneault H., Lefebvre R., Nastev $M$. Numerical simulation of the radius of influence for landfill gas wells // Vadose Zone Journal. - 2004. Vol. 3, Iss. 3. - P. 909-916.

2. Lei L., Bing L., Qiang X., Ying Z., Yongbo W. Estimation of radius of influence of pumping landfill gas // Journal of Chemical Industry and Engineering (China). - 2008. - Vol. 59, Iss. 3. - P. 751-755.

3. Feng S.-J., Zheng Q.-T. A two-dimensional gas flow model for layered municipal solid waste landfills // Computers and Geotechnics. - 2015. Vol. 63. - P. 135-145.
4. Lofy J.R. Zones of vacuum influence surrounding gas extraction well // Landfilling of Waste: Biogas. - London: E\&FN Spon, 1996. - P. 320-454.

5. Li Y., Batlle F., Carrera J., Lloret A. Gas flow to a vertical gas extraction well in deformable MSW landfills // Journal of Hazardous Materials. - 2009. Vol. 168, Iss. 2-3. - P. 1404-1416.

6. Tinet A.-J., Oxarango L. Stationary gas flow to a vertical extraction well in MSW landfill considering the effect of the mechanical settlement on hydraulic properties // Chemical Engineering Science. - 2010. - Vol. 65, Iss. 23. - P. 6229-6237.

7. PandolfiT., IsolaniR. Determinationofvacuum sphere of influence surrounding biogas extraction wells by a low expensive in situ methodology using a tracer gas // Thirteenth International Waste Management and Landfill Symposium: proceedings, Sardinia 2011 (S. Margherita di Pula, Calgari, Italy, October 3-7). - Padova: CISA Publisher, 2011. - 1 CD-disk. - Title from the paper.

8. Dillah D.D., McCarron G.P., Panesar B.S. Vertical landfill gas extraction well - The SCS model // SWANA's 30th Annual Landfill Gas Symposium: conference proceedings (Monterey, California, USA, March 4-8, 2007). - Silver Spring, Maryland: SWANA, 2007. - 1 CD-disk. - Title from the paper.

9. Куиый Д.В. Численное моделирование фильтрации биогаза и теплообмена в деформируемом теле полигона твердых бытовых отходов. Ч. II. Верификация модели и ее применение // Теплоэнергетика. - 2015. - № 7. - С. 36-43.

10.Yazdani R., Imhoff P., Han B., Mei C., Augenstein D. Quantifying capture efficiency of gas collection wells with gas tracers // Waste Management. - 2015. - Vol. 43. - P. 319-327.

11. Tian Y., Shi J., Wang J., Liang L. Simulation of the effective distance in horizontal direction from vertical well in landfill // The Electronic Journal of Geotechnical Engineering. - 2016. - Vol. 21, Iss. 4. - P. 1375 -1380. - Access mode: http://www.ejge. com/2016/Ppr2016.0124ma.pdf.

12. Young A., Gay N.J. Interactions between Gas Extraction Wells // Waste Management and Research. - 1995. - Vol. 13, Iss. 1. - P. 3-12.

13.Xin G.X., Song Y. Discuss on the design of 
MSW landfill gas collection project//3rd International Methane and Nitrous Oxide Mitigation Conference: conference papers (Hotel Kunlun, Beijing, China, November 17-21). - 2003. - Access mode: http:// www.coalinfo.net.cn/coalbed/meeting/2203/papers/ landfill/010.pdf (14.04.16). - Title from the paper.
14.Куц̧ый Д.В. Численное моделирование фильтрации биогаза и теплообмена в деформируемом теле полигона твердых бытовых отходов. Ч. I. Разработка модели // Теплоэнергетика. - 2015. - № 6. - С. 18-22. 


\section{OPTIMIZATION OF A DISTANCE BETWEEN WELLS OF LANDFILL GAS COLLECTION SYSTEM}

\section{Kutsyi D.V.}

Institute of Engineering Thermophysics of NAS of Ukraine, ul. Zhelyabova, 2a, Kyiv, 03057, Ukraine

The determination of the optimal distance between wells is a primary task of landfill gas collection system design. To solve this task, the principle which is based on maximization of the efficiency of landfill gas collection system and the flow rate in the well is offered. As the result of principle use it was determined that the optimal distance between wells is equal to double radius of influence. This distance is increase due to increase of extraction pressure in the well which effects on increasing of the efficiency of the landfill gas collection system.

Ref. 14, fig. 5.

Key words: extraction well, landfill gas, municipal solid waste, optimal distance, radius of influence.

1. Vigneault H., Lefebvre R., Nastev $M$. Numerical simulation of the radius of influence for landfill gas wells // Vadose Zone Journal. - 2004. Vol. 3, Iss. 3. - P. 909-916.

2. Lei L., Bing L., Qiang X., Ying Z., Yongbo W. Estimation of radius of influence of pumping landfill gas // Journal of Chemical Industry and Engineering (China). - 2008. - Vol. 59, Iss. 3. - P. 751-755. (China).

3. Feng S.-J., Zheng Q.-T. A two-dimensional gas flow model for layered municipal solid waste landfills // Computers and Geotechnics. - 2015. Vol. 63. - P. 135-145.

4. Lofy J.R. Zones of vacuum influence surrounding gas extraction well // Landfilling of Waste: Biogas. - London: E\&FN Spon, 1996. - P. 320-454.

5. Li Y., Batlle F., Carrera J., Lloret A. Gas flow to a vertical gas extraction well in deformable MSW landfills // Journal of Hazardous Materials. - 2009. Vol. 168, Iss. 2-3. - P. 1404-1416.

6. Tinet A.-J., Oxarango L. Stationary gas flow to a vertical extraction well in MSW landfill considering the effect of the mechanical settlement on hydraulic properties // Chemical Engineering Science. - 2010. - Vol. 65, Iss. 23. - P. 6229-6237.
7. PandolfiT., IsolaniR. Determination ofvacuum sphere of influence surrounding biogas extraction wells by a low expensive in situ methodology using a tracer gas // Thirteenth International Waste Management and Landfill Symposium: proceedings, Sardinia 2011 (S. Margherita di Pula, Calgari, Italy, October 3-7). - Padova: CISA Publisher, 2011. - 1 CD-disk. - Title from the paper.

8. Dillah D.D., McCarron G.P., Panesar B.S. Vertical landfill gas extraction well - The SCS model // SWANA's 30th Annual Landfill Gas Symposium: conference proceedings (Monterey, California, USA, March 4-8, 2007). - Silver Spring, Maryland: SWANA, 2007. - 1 CD-disk. - Title from the paper.

9. Kutsyi D.V. Numerical modeling of landfill gas and heat transport in the deformable MSW landfill body. Part 2. Verification and application of the model // Thermal Engineering. - 2015. - Vol. 62, Iss. 7. - P. 495-502.

10.Yazdani R., Imhoff P., Han B., Mei C., Augenstein D. Quantifying capture efficiency of gas collection wells with gas tracers // Waste Management. - 2015. - Vol. 43. - P. 319-327.

11.Tian Y., Shi J., Wang J., Liang L. Simulation of the effective distance in horizontal direction from vertical well in landfill // The Electronic Journal of Geotechnical Engineering. - 2016. - Vol. 21, Iss. 4. - P. 1375 -1380. - Access mode: http://www.ejge. com/2016/Ppr2016.0124ma.pdf.

12. Young A., Gay N.J. Interactions between Gas Extraction Wells // Waste Management and Research. - 1995. - Vol. 13, Iss. 1. - P. 3-12.

13.Xin G.X., Song Y. Discuss on the design of MSW landfill gas collection project//3rd International Methane and Nitrous Oxide Mitigation Conference: conference papers (Hotel Kunlun, Beijing, China, November 17-21). - 2003. - Access mode: http:// www.coalinfo.net.cn/coalbed/meeting/2203/papers/ landfill/010.pdf (14.04.16). - Title from the paper.

14.Kutsyi D.V. Numerical modeling of landfill gas and heat transport in the deformable MSW landfill body. Part 1. Development of the model // Thermal Engineering. - 2015. - Vol. 62, Iss. 6. - P. 403-407.

Получено 28.04.2016 Received 28.04.2016 